\title{
Simulation and experimental research on high-power microwave coupling with computer box
}

\author{
Liuhong Huang ${ }^{1,2}$ Jie Yang $^{2}$ Yaohui Zhang ${ }^{2}$ Cui Meng ${ }^{1}$ Yaobo Li $^{2}$ Zheng Pan $^{2}$ \\ ${ }^{1}$ Key Laboratory of Particle and Radiation Imaging, Department of Engineering Physics, Tsinghua University, Beijing, China \\ ${ }^{2}$ Research Institute for National Defense Engineering of Academy of Military Science PLA China, Beijing, China
}

\begin{abstract}
The electromagnetic coupling characteristics of a computer box under the high-power microwave (HPM) which includes the narrow-band and ultra-wideband HPM are studied. The simulations of radiation field in the computer box show that the optical drive and floppy disk drive are main channels through which the high-power microwave penetrates. The cut-off frequency of this corresponding dielectric waveguide and resonant frequency of computer box are the frequencies on which the coupling field is enhanced. The induced current on the cable show that the peak current value decreases with the increasing frequency. At the same time, the damage effect experiments of the computer under high-power microwave are carried out and the thresholds of three effect levels are summarized. The experimental results and the simulation are verified to some extent.
\end{abstract}

\section{INTRODUCTION}

The widespread application of large scale integrated circuits and multi-functional low-power chips makes the performance of computers better, while the risk of being interfered or damaged for computers increases. Therefore, it is necessary to study the electromagnetic damage characteristics of computers under the influence of external electromagnetic interference.

Taking the computer as an example, typical electromagnetic coupling approaches include aperture coupling through different types and numbers of apertures, conductive coupling through various power and signal cables. As long as the microwave energy coupled into computer box is enough large, the current or voltage induced on the circuit board exceed the threshold of sensitive components, which may cause interference on the electronic components and even damage the whole circuit system.

At present, there are many literatures on aperture coupling with metal cavity (Jedlicka, 2000; SIAH Eng Swee, 2003; Li Kai, 2013; Peng Qiang, 2013) and computer box is the common simulation object, while the optical drive and floppy disk drive are usually considered as complete cavity without holes (Chen Xiuqiao, 2004; Yao Jianming, 2005). This approach is not consistent with the reality. The waveguide models filled with internal dielectric medium for these two drives are more accurate. In this paper, the simulative model of a kind of computer box is established and the electromagnetic simulation software FEKO based on the method of moment (MoM) is used to simulate the radiation field of internal space and the induced current on the cable. Furthermore, the experimental results of the high-power microwave (HPM) radiating on the computer are given and compared with the simulation.

\section{SIMULATION RESEARCH}

Taking the IBM 8179 desktop computer box as the simulation object which is shown in Figure 1, this model is established by using FEKO and has some components such as a power supply, an optical drive, a floppy disk drive, a hard disk and a motherboard, et al. According to the real computer box, there are many different numbers, sizes and shapes of the air vents in the front, back and side panels. However, the vents which are less than one tenth of the wavelength $(3 \mathrm{~mm})$ are omitted in order to simplify the model and save the computational resources (the max frequency is $10 \mathrm{GHz}$ ). At the same time, a single conductive cable with length of $1 \mathrm{~m}$ is inserted into the computer box. The electromagnetic parameters of each part are shown in Table 1. 


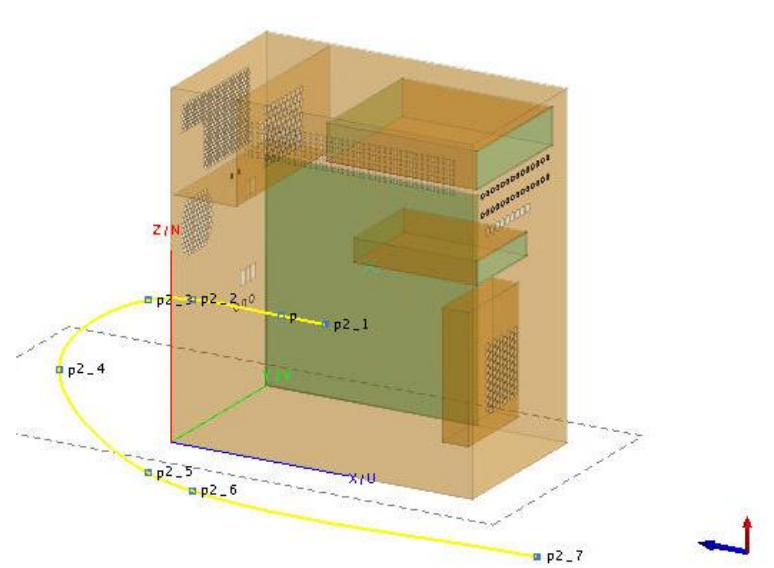

Figure 1. Model of the computer box with a cable

Table 1. Electromagnetic parameters of the computer box

\begin{tabular}{|c|c|c|c|c|}
\hline Name & Size $(\mathbf{m m})$ & $\boldsymbol{\varepsilon}_{\boldsymbol{r}}$ & $\boldsymbol{\mu}_{\boldsymbol{r}}$ & $\boldsymbol{\sigma}$ \\
\hline $\begin{array}{c}\text { Computer } \\
\text { box }\end{array}$ & $340 \times 190 \times 370$ & 1.0 & 1.0 & $3 \times 10^{7}$ \\
\hline Power shell & $70 \times 186 \times 110$ & 1.0 & 1.0 & $3 \times 10^{7}$ \\
\hline Optical drive & $170 \times 150 \times 40$ & 1.0 & 1.0 & $3 \times 10^{7}$ \\
\hline Floppy drive & $140 \times 100 \times 25$ & 1.0 & 1.0 & $3 \times 10^{7}$ \\
\hline
\end{tabular}

\begin{tabular}{|c|c|c|c|c|}
\hline Hard disk & $30 \times 100 \times 140$ & 1.0 & 1.0 & $3 \times 10^{7}$ \\
\hline $\begin{array}{c}\text { Motherboar } \\
\text { d }\end{array}$ & $240 \times 1 \times 240$ & 2.5 & 1.0 & 0 \\
\hline Vents & $1 \times 2$ & 1.0 & 1.0 & 0 \\
\hline Cable & $\begin{array}{c}\text { Diameter }=2 \mathrm{~mm} \\
\text { Length }=1000 \mathrm{~mm}\end{array}$ & 1.0 & 1.0 & $3 \times 10^{7}$ \\
\hline
\end{tabular}

\subsection{Electric Field Distribution}

The coupling characteristics of the box under the incident narrow-wideband HPM are calculated. In order to compare with the later experimental results, the narrowband HPM is divided into four bands of $\mathrm{L}(1.3 \mathrm{GHz}), \mathrm{S}$ $(2.85 \mathrm{GHz}), \mathrm{C}(5.65 \mathrm{GHz})$ and $\mathrm{X}(9.34 \mathrm{GHz})$. We can use plane wave with these frequencies as the incident narrow-band HPM to simply the simulation.

As shown in Figure 2 (a) $($ f), these give the electric field amplitude on the central plane of the box. The plane wave is incident perpendicularly from the front of the box. The polarization of the electric field is parallel to the $\mathrm{z}$-axis with the amplitude of $1 \mathrm{~V} / \mathrm{m}$. Figure 3 shows the electric field amplitude on the geometric centre of the box.

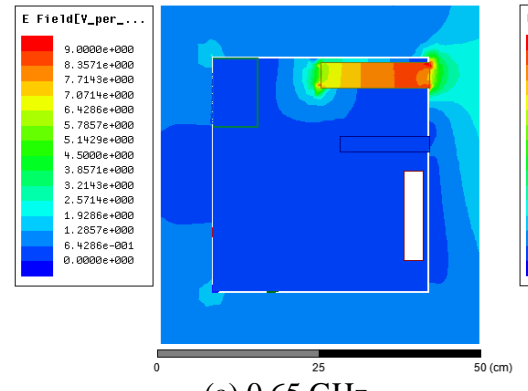

(a) $0.65 \mathrm{GHz}$

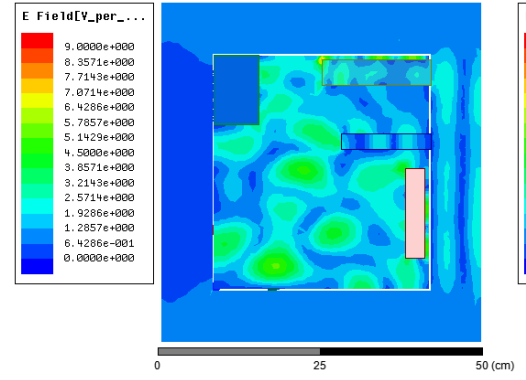

(d) $2.85 \mathrm{GHz}$
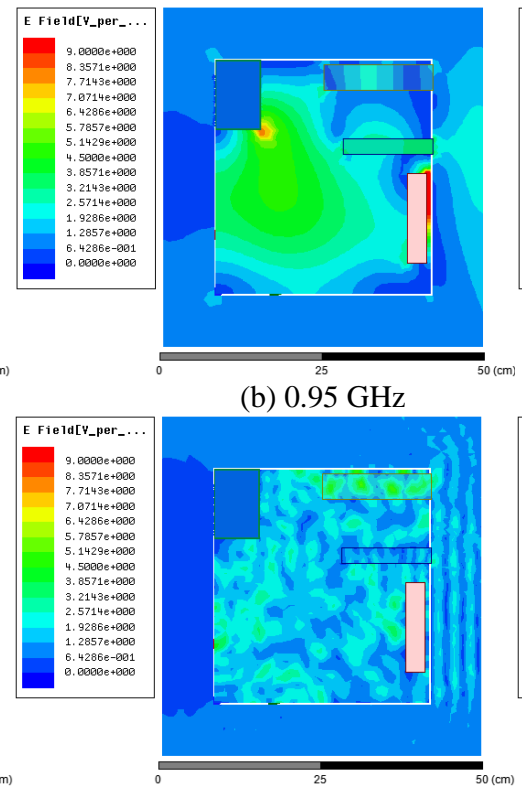

(e) $5.65 \mathrm{GHz}$
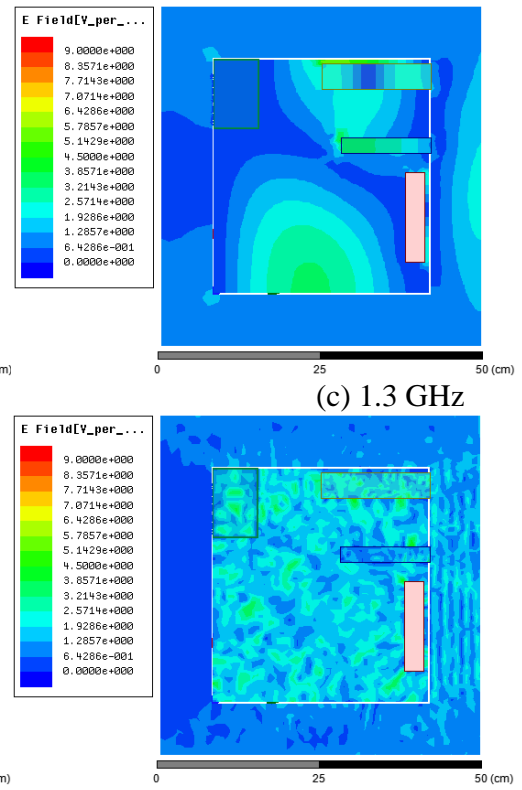

(f) $9.34 \mathrm{GHz}$

Figure 2. Electric field amplitude at different frequencies 


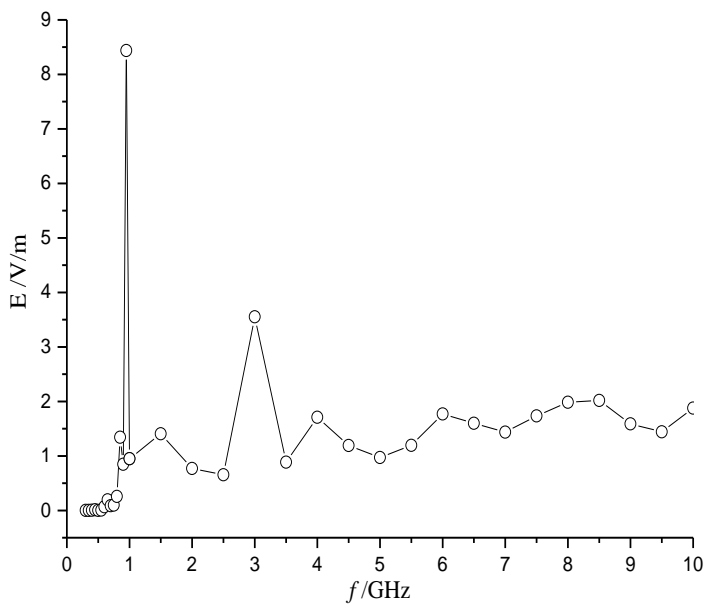

Figure 3. Electric field amplitude on the geometric centre of the box

According to the results of Figure 2 and Figure 3, the following conclusions can be drawn:

(1) Optical drive and floppy drive are the main ways in which an external electromagnetic field couples into computer box. The higher the frequency is, the easier it is to couple in. In the simulation of this paper, these two drives are all dielectric rectangular waveguide models. According to the cut-off frequency formula (Zhou Bihua, 2006), they are $0.63 \mathrm{GHz}$ and $0.95 \mathrm{GHz}$. This is consistent with the result shown in Figure 2 (a) and (b) that the electromagnetic wave starts to couple into the box through these two drives at $0.65 \mathrm{GHz}$ and $0.95 \mathrm{GHz}$ respectively. With the increase frequency of the incident wave, the vents of the front panel gradually become the passageway into computer box and the electromagnetic field distribution inside the box gradually becomes uniform.

(2) In terms of the geometric centre of the box, the shielding effectiveness at $0.95 \mathrm{GHz}$ and $3 \mathrm{GHz}$ is relative low in the calculated frequency range, with the electric field is $8.44 \mathrm{~V} / \mathrm{m}$ at $0.95 \mathrm{GHz}$ while the lowest shielding effectiveness is $-18.5 \mathrm{~dB}$. The first reason as mentioned above is that the frequency $0.95 \mathrm{GHz}$ is exactly the cutoff frequency of the floppy drive. The second reason, meanwhile, is that this frequency is close to one of the resonant frequencies of the box (the first resonant frequency above $0.3 \mathrm{GHz}$ is $0.6 \mathrm{GHz}$ and the second is $0.9 \mathrm{GHz}$ ), which means it's easy to generate strong coupling.

\subsection{The Induced Current}

The induced current on the cable which is inserted into computer box is simulated. The cable is a single conductor with two open ends and averagely divided into 195 segments which are sequentially numbered from the beginning outside the box to the ending within the box.

The waveform formula of the incident ultra-wideband HPM is differential Gauss pulse (The amplitude have been normalized):

$$
E_{i}(t)=\left(t-t_{0}\right) \exp \left[-\frac{4 \pi\left(t-t_{0}\right)^{2}}{\tau^{2}}\right]
$$

According to the highest pulse frequency of $1.5 \mathrm{GHz}$, the pulse width $\tau=1.3 \mathrm{~ns}, \mathrm{t}_{0}=2 \mathrm{~ns}$. Figure 4 shows the waveforms of three electric field components at the centre point of the box. It can be seen that under the excitation of a differential Gaussian pulse with polarization direction of Ez, three waveforms fluctuate violently and take a relative long time to decay. Figure 5 shows the induced current on the start point (No. 1) and centre point (No. 98) of the cable. The current on the centre point is much higher and fluctuate because of two opening ends of the cable.

The distribution of induced current along the cable under four different frequencies which can be seen as the narrow-band HPM is shown in Figure 6. It can be seen that the internal induced current on the cables is generally higher than the external, which is influenced by the increasing field strength in the box. In addition, with the increasing frequency of incident wave, the peak value of induced currents on the cable are reduced from $0.56 \mathrm{~mA}$ to $0.13 \mathrm{~mA}$, which indicates that the low-frequency coupling of electromagnetic wave on the cable is more obvious.

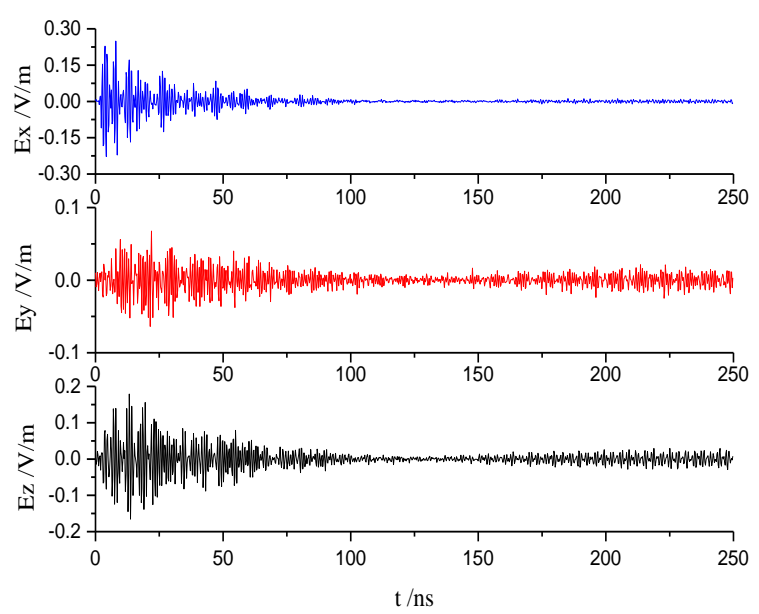

Figure 4. Electric field components at the centre point of computer box

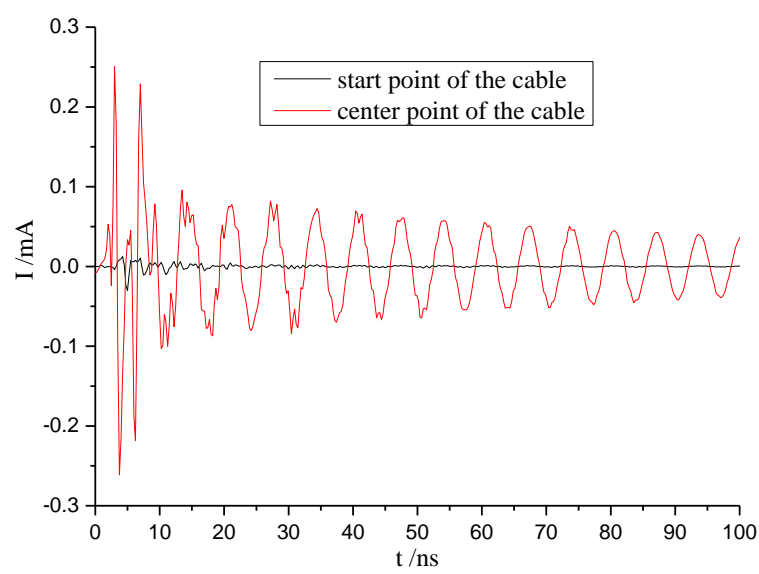

Figure 5. The induced current at two points on the cable 


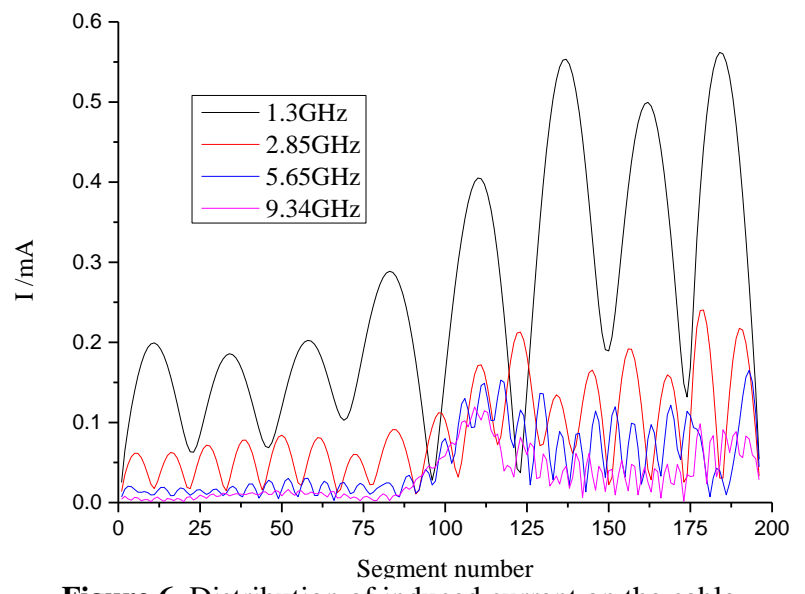

Figure 6. Distribution of induced current on the cable

\section{EXPERIMENTAL RESEARCH}

The damage effect tests of HPM on the IBM 8179 are carried out. The test system is composed of pulse radiation source (including the narrow-band and ultra- wideband HPM), transmitting and receiving antennas, microwave anechoic chamber and measuring system. The layout of the damage effect test is shown in Figure 7. The narrow-band HPM is divided into four bands of $\mathrm{L}, \mathrm{S}, \mathrm{C}$ and $\mathrm{X}$. The waveform of the ultra-wideband HPM is differential Gaussian pulse which has been used in the simulation. Some interference or damage on the computer which reflect on the screen can been shown in Figure 8 .

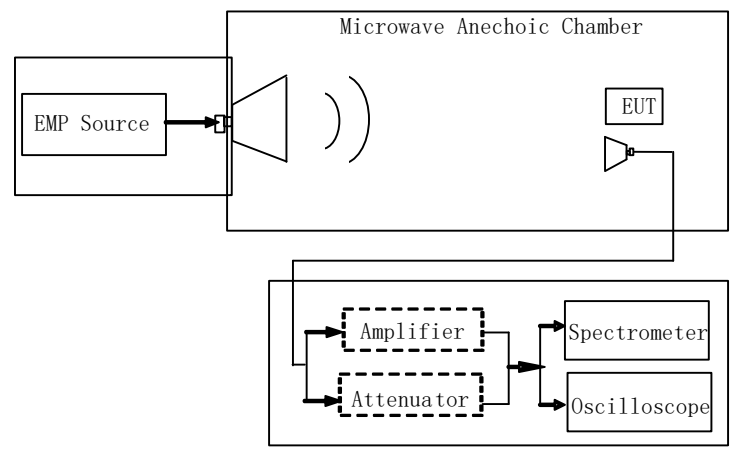

Figure 7. Layout of the damage effect test on the EUT

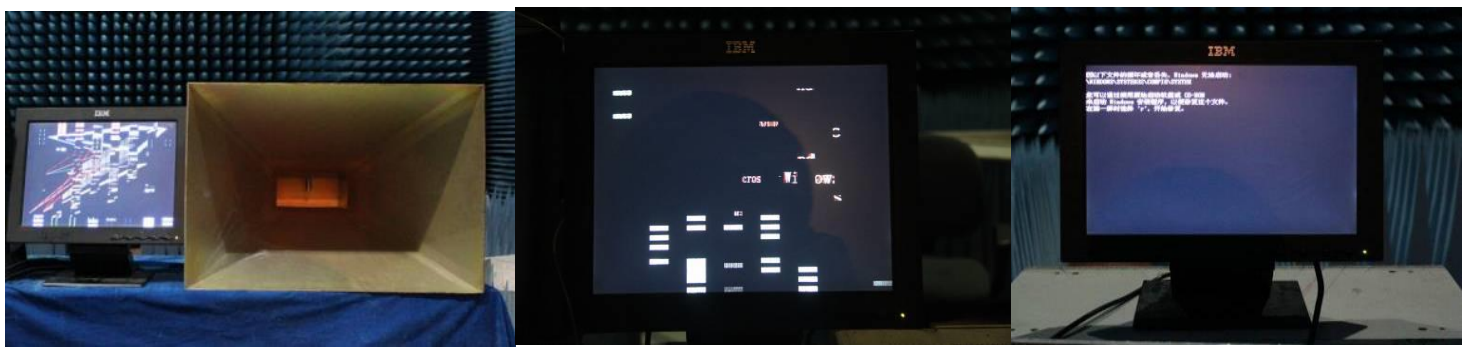

(a) interfere the screen

(b) lost some displayed information

(c) ruin some system files

Figure 8. Some interference or damage on the computer which reflect on the screen

Table 2. Effect threshold of the computer host

\begin{tabular}{|c|c|c|c|c|c|}
\hline \multirow{2}{*}{ Effect level } & \multicolumn{4}{|c|}{$\begin{array}{c}\text { Narrow-band HPM } \\
\left(\mathbf{J} / \mathbf{m}^{2}\right)\end{array}$} & $\begin{array}{c}\text { Ultra-wideband } \\
\text { HPM } \\
(\mathbf{V} / \mathbf{m})\end{array}$ \\
\cline { 2 - 6 } & $\mathbf{L}$ & $\mathbf{S}$ & $\mathbf{C}$ & $\mathbf{X}$ & $2.08 \sim 10.49$ \\
\hline Interference & $0.01 \sim 0.17$ & $0.02 \sim 0.20$ & $0.32 \sim 1.74$ & $2069.87 \sim 4981.09$ \\
\hline Disturbance & $0.20 \sim 0.95$ & $0.12 \sim 1.21$ & $4.66 \sim 28.33$ & $17.73 \sim 115.08$ & $4823.38 \sim 12618.31$ \\
\hline Damage & $3.47 \sim 26.23$ & $3.36 \sim 19.94$ & $30.62 \sim 148.99$ & $93.68 \sim 400.58$ & $13227.04 \sim 34591.81$ \\
\hline
\end{tabular}

In order to obtain the thresholds of three levels of damage (interference, disturbance and damage), a huge number of tests with different pulse widths and repetition frequencies (for narrow-band HPM), different repetition frequencies (for ultra-wideband HPM), different polarizations and radiation angles are conducted. After analysing the large number of experimental data, the relationship between the power density or peak field strength with the damage effect is established. The thresholds of computer host are shown in Table 2. Due to the limitation of the experimental conditions, the electric field in the centre of the box and the induced current on the cable cannot be measured. Therefore, the test results cannot be compared quantitatively with the simulation results. However, the following conclusions can be verified to some extent with simulation results.

(1)Experimental results show that the threshold is the lowest when the incident wave is perpendicular to the front face of computer box. The threshold when to the side-face of the box is the highest. Simulation results of the electric field in the box validate these rules.

(2)The threshold is the lowest when the incident frequency is $\mathrm{S}(2.85 \mathrm{GHz})$ among the four frequency of narrow-band HPM. As shown in Table 2, the maximum energy density required for damage in $\mathrm{S}$ is $19.94 \mathrm{~J} / \mathrm{m}^{2}$. The required energy density for $\mathrm{L}, \mathrm{C}$ and $\mathrm{X}$ is a maximum of $26.23 \mathrm{~J} / \mathrm{m}^{2}, 148.99 \mathrm{~J} / \mathrm{m}^{2}$ and $400.58 \mathrm{~J} / \mathrm{m}^{2}$. The simulation result shown in Figure 3 also reflects that 
the centre point is the strong coupling point at $3 \mathrm{GHz}$ and the electric field in the vicinity is relatively high.

(3)The results of Table 2 also show that the thresholds of three effect levels increase with the frequency. In addition, the effect of cable coupling is greater than aperture coupling when the cable is inserted into computer box. The simulation shows that the higher the frequency, the easier the aperture coupling and the electromagnetic field distribution is relatively more uniform, while the amplitude of the electric field just shows small fluctuations. The induced current on the cable decreases with increasing frequency, indicating that a stronger coupling of low frequency electromagnetic wave. Therefore, the synthetic analysis of aperture and cable coupling can deduce the test results that the effect threshold increases with increasing frequency.

(4)Table 2 also shows that the threshold of ultrawideband HPM is much higher than narrow-band HPM. Comparing the results of Figure 3 and Figure 5, both of which incident waves are normalized, it is obvious that the former has higher spatial electric field than the latter

\section{CONCLUSIONS}

In this paper the coupling and damage effect of HPM on the computer are studied. The simulations focus on the internal radiation field and induced currents on the cable. In order to obtain the threshold of three damage levels of computer under the narrow-band and ultra-wideband HPM, much experimentation are carried out. At the same time, the mutual verification of experimental conclusions and simulation results is achieved. The next research will focus on the simulation of HPM coupling on the components and circuit board inside the box.

\section{REFERENCES}

1. Jedlicka, 2000. Coupling through tortuous path narrow slot apertures into complex cavities. In IEEE Trans. Antenna Propagation.

2. SIAH Eng Swee, SERTEL Kubilay, VOLAKIS John L, et al., 2003. Coupling studies and shielding techniques for electromagnetic penetration through apertures on complex cavities and vehicular platforms. In IEEE Trans. on EMC.

3. Li Kai, Wei Guanghui, Pan Xiaodong, et al., 2013. Research of the shielding effectiveness of a rectangular metallic enclosure with an aperture. In Journal of Microwaves.

4. Peng Qiang, Zhou Dongfang, Hou Deting, et al., 2013. Shielding effectiveness analysis of rectangular cavity with aperture by modification and expansion of transmission line method. In High Power Laser And Particle Beams.

5. Chen Xiuqiao, Hu Yihua, Zhang Jianhua, et al., 2004 Simulation of electromagnetic pulse coupling with computer box. In Journal of System Simulation.

6. Yao Jianming, Wu Jianjiang, Xie yongjun, et al., 2005. Study on shielding effect of electronic equipments with built-in PCB circuit. In Journal of Microwaves.

7. Zhou Bihua, Gao Cheng, Shi Lihua, et al., 2006. The electromagnetic pulse protective design of the civil engineering, National Defense Industry Press. Beijing. 